Introduction As a consequence of increased SLE patients survival, patients with long disease duration represent a significant proportion of our cohorts. This study aims to evaluate health resource use and the 6 months costs in patients with SLE with long disease duration.

Methods The economic evaluation was performed in terms of cost-of-illness analysis as part of a larger study enrolling SLE patients with at least 15 years of disease duration regularly followed at our unit. At enrollment, the following information were collected: disease activity (SLEDAI), organ damage (SLICC-DI score), comorbidities, treatment patterns; in addition to clinical data, patients were required to complete an ad-hoc questionnaire for the collection of facts relevant for the estimation of the economic dimension and covering the previous six-months. Such a time frame was considered to be appropriate as recall period. Direct health (drugs, hospitalizations, emergency visits, specialists visits, laboratory tests and instrumental examination) and non-health costs (transportation and accommodation) as well as indirect costs because of productivity loss were estimated.

Results 51 adult patients with long disease duration were recruited $(98 \%$ female, mean age $49 \pm 11$ years, median disease duration 17 years, IQR 15-23). Median (IQR) SLEDAI score was $2(0-4)$, median SLICC-DI was $1(0-2)$. The median (IQR) direct health costs per patients over the previous 6 months resulted $410 €(201-1687)$; indirect costs because of productivity lost were $130 €(0-356)$. The median overall cost to the Society was $473 €(327-2148)$; the presence of comorbid conditions resulted associated with higher overall cost for the Society (552€ [327-1807] vs 264€ [94-1164] p=0.046); disease activity and damage at enrollment were not associated with costs increase in this cohort.

Conclusions This cohort of patients with long lasting disease is characterised by low disease activity and mild organ damage; in this setting, the disease burden on the single patient and family is significant and the costs to the Society are influenced by the presence of comorbidities.

\begin{tabular}{|c|c|c|c|}
\hline \multicolumn{4}{|c|}{ Abstract PS8:161 Table 1} \\
\hline & 0 & 1 & 2 \\
\hline $\begin{array}{lr}\text { Hospitalizations } & \text { due } \\
\text { to SLE } & \text { or } \\
\text { complications } & \end{array}$ & $40(78.4 \%)$ & $10(19.6 \%)$ & $1(2 \%)$ \\
\hline \multirow[t]{2}{*}{ ER visits } & $45(88.2 \%)$ & $6(11.7 \%)$ & 0 \\
\hline & Median & $\begin{array}{c}\text { Interquartile } \\
\text { range }\end{array}$ & \\
\hline Specialist visit & 1 & $1 \cdot 3$ & \\
\hline Instrumental exams & 2 & $1 \cdot 3$ & \\
\hline Laboratory exams & 2 & 1.4 & \\
\hline
\end{tabular}

\section{PS8:162 DISEASE PATTERN OF MALE LUPUS}

N Klyukvina, E Nasonov. V.A.Nasonova Research Institute of Rheumatology, Moscow, Russia

\subsection{6/lupus-2018-abstract.205}

Introduction Systemic lupus erythematosus (SLE) is a multisystem autoimmune disease that occurs primarily in women $(9: 1$ compared to men). Available data argue that gender and sex hormones modify susceptibility to, and expression of SLE.
Subjects and methods One hundred and fifty male patients (pts) aged 15 to 64 years (mean age 30, 5 y) with a valid diagnosis of SLE who had been followed up for 15 years were assessed. Results: The mean age at onset was 20 year (range 6-62 years). The distribution of disease onset by decade of age was following: between the ages of 6-20 y - 80 pts (53\%), 21-40 y - 49 pts (33\%), 41-64 y - 21 pts (14\%). Mean duration from the onset to time of diagnosis valid SLE was 12 month (range 1-336). 77 pts were diagnosed during first year from disease onset. 35 pts had delay of true diagnosis for 5 and more years. The most common manifestations for men at disease onset were arthritis (50\%), renal disease $(16,7 \%)$ and haematological abnormalities $(23,3 \%)$. During follow-up we observed malar rash (58\%), arthritis $(73,3 \%)$, renal disease $(54,7 \%)$, CNS involvement $(49,4 \%)$, photosensitivity (51,3\%), discoid lupus (14\%), mucosal ulcers (28\%), serositis $(32,7 \%)$, haematological (72\%) and immunological $(93,3 \%)$ abnormalities.

41 male pts $(27,3 \%)$ satisfied classification Sapporo criteria of definite antiphospholipid syndrome (APS)/Vascular thrombosis developed in 41 pts: 26 pts had only venous thrombosis, 8 pts - merely arterial thrombosis, 7 pts $-\mathrm{a}+\mathrm{v}$ thrombosis. Pts with APS had higher frequency of livedo reticularis (13 pts, 31,7\%), pulmonary hypertension (8 pts, 19,5\%) and heart valve disease (20 pts, 48,8\%) in comparison to pts without APS (11,9\%, 6,5\%, 16,6\% respectively).

Conclusion The present investigation do not confirm older age at disease onset among men with SLE: one half of our pts were younger $20 \mathrm{y}$ at disease onset. We found high frequency of arthritis, renal disease and neuropsychiatric manifestations over the course of illness. CNS involvement was the rare initial symptom. Men with SLE often had delay of valid diagnosis of SLE. We observed high incidence of definite APS in men with SLE.

\section{PS8:163 CHARACTERISTICS AND OUTCOME OF LUPUS NEPHRITIS IN MOROCCO}

I Haddiya. Faculty of Medicine, University Mohamed First, Oujda, Morocco

\subsection{6/lupus-2018-abstract.206}

There is wide variation in clinical presentation and outcome of lupus nephritis (LN) among different ethnic groups. Few data for LN exist on North Africans, especially those from Morocco. The aim of our study was to review retrospectively the features and outcome of LN in Moroccan patients.

Patients and methods We performed a multicentric retrospective study. A total of 209 patients with LN were included. All patients met American Rheumatism Association criteria. LN was classified according to the International Society of Nephrology/Renal Pathology Society classification. We adopted previously defined outcome criteria for LN.

Results There were 185 females and 24 males, with a mean age of 27.2 years. At first presentation, we noted hypertension in $31 \%$, hematuria in $72 \%$, nephrotic syndrome in $47 \%$, and renal failure in $64 \%$ of cases. Renal biopsy revealed predominant proliferative classes in more than $80 \%$ of patients. Patients received different regimens mainly based on intravenous cyclophosphamide and Mycophenolate. After a mean follow-up of 22 months, remission occurred in 46.7\%, relapses in $81 \%$, end-stage renal failure in $24 \%$, and death in $13 \%$ of 
cases. Infection and neurological and cardiovascular diseases were the most frequent causes of death.

Conclusion LN seems to be severe in our study, with a predominance of proliferative forms, severe renal manifestations, and poor renal and overall survival.

\section{PS8:164 COMPARISON OF CLINICAL AND SEROLOGICAL FEATURES OF JUVENILE AND ADULT-ONSET NEUROPSYCHIATRIC LUPUS IN SPANISH PATIENTS}

WA Sifuentes Giraldo, AL Boteanu, SGarrote Corral, A García Fernández, MJ García Villanueva, ML Gámir Gámir, AZea Mendoza. Hospital Universitario Ramón y Cajal, Madrid, Spain

\subsection{6/lupus-2018-abstract.207}

Background Neuropsychiatric (NP) manifestations are a main cause of morbidity and mortality in juvenile-onset systemic lupus erythematosus (jSLE). Some studies suggest that they are more frequent and severe in jSLE than in adult-onset SLE (aSLE).

Objectives To compare clinical and serological profiles of paediatric and adult patients with neuropsychiatric lupus (NPSLE) treated in a Spanish tertiary centre.

Methods We performed a retrospective study of patients with jSLE (onset age: 0-18 y) and aSLE (onset age: $>18$ y) seen in our centre during the period 1988-2016. ACR's case definitions were used to identify NPSLE manifestations. Demographics, clinical and serological data were obtained from their medical records.

Results 69 patients with NPSLE were included (aSLE 41, jSLE 28), most of them Caucasians (92\%). Mean age at diagnosis was 36.4 y (range: 19-68) in adults and 13.9 y (range: 8-18) in children. The proportion of males was higher in the latter group. Mean disease duration was significantly greater in adults, as well as time from SLE diagnosis to NP manifestation onset, although without significant difference (comparison of groups is presented in the table). Central NP manifestations were more frequent (aSLE 93\%, jSLE 96\%) than peripheral manifestations (aSLE 12\%, jSLE 11\%). The most common manifestations in aSLE were headache (29\%), cerebrovascular disease $(27 \%)$ and seizures (17\%), whereas in jSLE were seizures (46\%), headache (29\%) and mood disorder/depression (25\%). A significant group of patients presented 2 or more central manifestations during follow-up (aSLE 32\%, jSLE 41\%); mean number of manifestations was 1.36 (range: 1-3) in adults and 1.44 (range: 1-4) in children. jSLE patients with developed lupus nephritis more frequently, as well as higher anti-DNA antibodies titres, increased erythrosedimentation rate (ESR) and hypocomplementemia. Mortality occurred in 2 cases of aSLE and 2 jSLE.

Conclusions Our results corroborate that juvenile patients with NPSLE present higher disease activity compared to adults. There was no significant diference in time from SLE diagnosis to NP manifestation onset, but tended to be shorter in jSLE. The spectrum of NPSLE was varied both groups and an important proportion developed 2 or more manifestations. Mortality continues to be important in NPSLE in both age groups.

\section{PS8:165 NEUROPSYCHIATRIC DAMAGE IN DECEASED PATIENTS WITH SYSTEMIC LUPUS ERYTHEMATOSUS}

${ }^{1}$ I Padjen, ${ }^{2} \mathrm{M}$ Erceg, ${ }^{2} \mathrm{M}$ Cerovec, ${ }^{1} \mathrm{M}$ Mayer, ${ }^{1} \mathrm{R}$ Stevanovic, ${ }^{1} \mathrm{~B}$ Anic. ${ }^{1}$ Division of Clinical Immunology and Rheumatology, UHC Zagreb, University of Zagreb, School of Medicine, Zagreb, Croatia; ${ }^{2}$ Croatian Institute of Public Health, Zagreb, Croatia

\subsection{6/lupus-2018-abstract.208}

Purpose Neuropsychiatric damage (ND) is a major determinant of morbidity in SLE. We analysed ND in a group of deceased SLE patients and identified features associated with ND.

Methods We retrospectively analysed 90 patients (68 females) deceased during 2002-2011. All patients fulfilled at least 4 classification criteria of the ACR. We identified patients with $\mathrm{ND}$, as defined by the SLICC/ACR damage index, and its components: cognitive impairment/major psychosis (CIMP), seizures, cerebrovascular accident (CA), cranial/peripheral neuropathy and transverse myelitis (TM). Following variables were compared between patients with and without ND (ND +and ND, respectively): demographics, ACR criteria at diagnosis and cumulatively at death (available at diagnosis for

\begin{tabular}{|c|c|c|c|}
\hline & Juvenile NPSLE & Adult NPSLE & p-value \\
\hline $\mathrm{N}^{\circ}$ of patients & $28(41 \%)$ & $41(59 \%)$ & - \\
\hline Women:men & $20: 8$ & $39: 2$ & $0.0060 *$ \\
\hline Time of disease (months) & 19.8 & 232.5 & $0.0001 *$ \\
\hline NP manifestations at onset & $7(25 \%)$ & $11(27 \%)$ & 0.8651 \\
\hline Time from diagnosis to NP manifestation (months) & 42.4 & 87.1 & 0.1268 \\
\hline Lupus nephritis & $16(57 \%)$ & $9(22 \%)$ & $0.0028 *$ \\
\hline Antiphospholipid syndrome & $5(18 \%)$ & $10(24 \%)$ & 0.5182 \\
\hline ANA $\geq 1 / 1280$ & $8(29 \%)$ & $11(27 \%)$ & 0.8736 \\
\hline Anti-DNA ab (IU/ml) & 178.9 & 39.4 & $0.0005 *$ \\
\hline Anti-Ro/SSA ab & $10(36 \%)$ & $17(41 \%)$ & 0.6308 \\
\hline Anticardiolipin ab & $4(14 \%)$ & $10(24 \%)$ & 0.3054 \\
\hline Anti- 32 glycopotein I ab & $5(18 \%)$ & $7(17 \%)$ & 0.9328 \\
\hline Lupus anticoagulant & $8(29 \%)$ & $10(24 \%)$ & 0.6977 \\
\hline Cryoglobulins & $6(21 \%)$ & $3(7 \%)$ & 0.0874 \\
\hline $\operatorname{ESR}(\mathrm{mm} / \mathrm{h})$ & 53.8 & 35.7 & $0.0199 *$ \\
\hline CRP mg/1 & 4.6 & 4.7 & 0.9687 \\
\hline C3 low $(<80 \mathrm{mg} / \mathrm{dl})$ & $22(79 \%)$ & $16(39 \%)$ & $0.0012 *$ \\
\hline C4 low $(<16 \mathrm{mg} / \mathrm{dl})$ & $22(79 \%)$ & $13(32 \%)$ & $0.0001 *$ \\
\hline
\end{tabular}

